\title{
Stochastic Resonance in Neuron Models
}

\author{
André Longtin ${ }^{1}$
}

\begin{abstract}
Periodically stimulated sensory neurons typically exhibit a kind of "statistical phase locking" to the stimulus: they tend to fire at a preferred phase of the stimulus cycle, but not at every cycle. Hence, the histogram of interspike intervals (ISIH), i.e., of times between successive firings, is multimodal for these neurons, with peaks centered at integer multiples of the driving period. A particular kind of residence time histogram for a large class of noisy bistable systems has recently been shown to exhibit the major features of the neural data. In the present paper, we show that an excitable cell model, the FitzhughNagumo equations, also reproduces these features when driven by additive periodic and stochastic forces. This model exhibits its own brand of stochastic resonance as the peaks of the ISIH successively go through a maximum when the noise intensity is increased. Further, the presence of a noise-induced limit cycle introduces a third time scale in the problem. This limit cycle is found to modify qualitatively the phase-locking picture, e.g., by suppressing certain peaks in the ISIH. Finally, the role of noise and possibly of stochastic resonance (SR) in the neural encoding of sensory information is discussed.
\end{abstract}

KEY WORDS: Stochastic resonance; neuron models.

\section{INTRODUCTION}

This paper examines the connection between sensory neuron activity and the dynamics of bistable and excitable systems in the presence of noise and periodic forcing. Our theoretical study is motivated by experimental studies of the firing activity of neurons in various sensory modalities (vision, audition, touch, etc.). ${ }^{(2-5)}$ The goal of those studies was to shed light on the sensory encoding mechanism used by the nervous system. The activity of these neurons in the presence of a periodic stimulus (e.g., a mechanical

\footnotetext{
${ }^{1}$ Département de Physique, Université d'Ottawa, 150 Louis Pasteur, Ottawa, Canada, K1N 6N5 and Complex Systems Group, Theoretical Division B213, and Center for Nonlinear Studies, Los Almos National Laboratory, Los Alamos, New Mexico 87545.
} 
vibration or a pure acoustic tone) exhibits various degrees of phase locking to the stimulus, depending on the stimulus characteristics. The firing occurs near a preferred phase of the external stimulus, but there can be a random number of cycles "skipped" between two successive firings. This leads to a multimodal distribution of interspike intervals (ISIs) with peaks centered around the integer multiples of the period of the stimulus (see Fig. 1). Apart from the phase preference, the firing appears aperiodic. A return map of successive ISIs reveals a lattice structure which appears symmetric about the 45-deg line, an indication that there is no apparent correlation between successive ISIs. Connecting the successive points also does not reveal any obvious deterministic pattern.

It has been shown recently that the histogram of interspike intervals (ISIH) is similar in many respects to that of residence times in bistable system. ${ }^{(1,6,7)}$ These studies demonstrate that model bistable systems can easily and robustly reproduce the neural data, provided little or no switching between the wells occurs in the absence of stochastic driving. This finding leads to the exciting possibility that stochastic resonance, which is an amplification of the signal by the noise, could play a role in the encoding process. While various forms of bistability have been exhibited by neurons as well as by neuron models (see Sections 2 and 3), the most generic behavior of neurons is best characterized as "excitable" rather than bistable: when a certain threshold state (membrane voltage) is reached, there occurs a large excursion in the state variable, followed by a recovery to a resting state (Section 4). The large excursion-reset event occurs on a
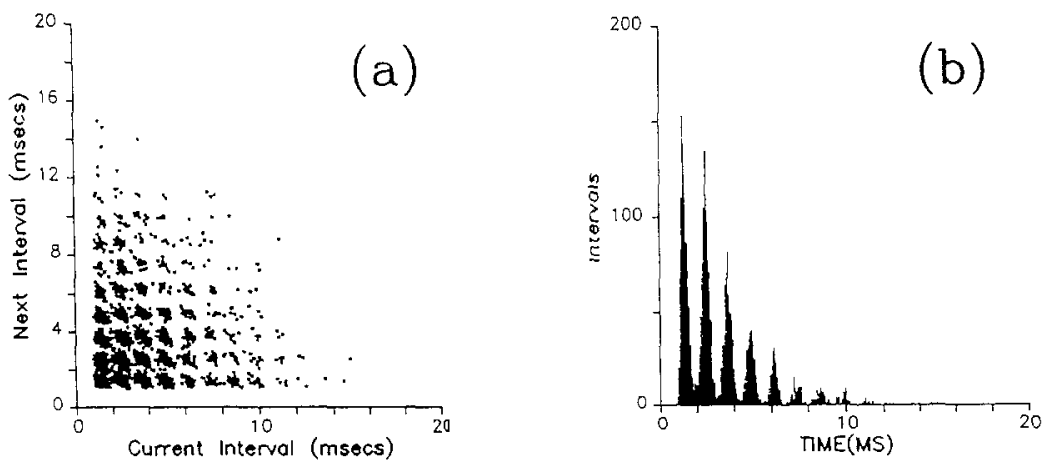

Fig. 1. Interspike interval data from extracellular recording of cat auditory nerve fibers. The stimulus was a pure $800-\mathrm{Hz}$ tone. (a) Return map (or joint interval histogram) of the interspike interval times series. The stimulus intensity is $60 \mathrm{~dB}$. (b) Interspike interval histogram (ISIH) for the same data as in (a). This is a plot of the number of interval events versus the interval duration. 
very fast time scale, and appears as a spike in the time course of the state variable. The obvious questions raised in this context and addressed in this paper are: (1) under what conditions can a periodically forced excitable neuron model exhibit the skipping phenomenon, and (2) what is the equivalent of "stochastic resonance" in such an excitable system which is not bistable?

The outline of the paper is as follows. In Section 2, we give some background on the neural data of interest and give a brief review of previous modeling efforts. Section 3 presents numerical simulations of a simple bistable model to illustrate how this class of models captures the main features of the neural data. A generic excitable cell model is then shown in Section 4 to also account for these features. This physiologically more appealing model is then studied from the point of view of stochastic resonance in Section 5, and a discussion follows in Section 6.

\section{BACKGROUND}

Physical stimuli produce time-dependent ionic currents in the neuron membrane, which give rise to action potentials ("firings" or "spikes") when the membrane voltage is sufficiently depolarized. Neurons in the auditory fiber of the squirrel monkey, ${ }^{(2)}$ in mechanoreceptive afferents of the hand of the macaque monkey, ${ }^{(3)}$ and in the primary visual cortex of the cat ${ }^{(5)}$ are among the classes of cells which exhibit multimodel ISIHs when subjected to a periodic stimulus (see Fig. 1). The peaks of these ISIHs are located at integer multiples of the driving period $T$ and, except for the first few peaks, the envelope of the peaks decays exponentially. The exception is due to the fact that the mode of the ISIH shifts away from the first peak at high frequency and/or low amplitude of the stimulus. The ISI first-return map (Fig. 1) suggest that there is little if any correlation between successive firing times, although sophisticated data analysis techniques have uncovered clustering and long-term correlations between the spikes in auditory data. ${ }^{(8)}$ Recent work, ${ }^{(1)}$ aimed at elucidating the simplest physical mechanisms which can exhibit the basic features of the data, has shown that this can be achieved by a wide class of periodically and stochastically driven bistable systems. This result hinges on the careful association of "residence time in wells" with the ISI measured in the physiological systems (see Section 3).

The problem of phase locking of noisy bistable systems to a periodic input has received attention especially in the context of stochastic resonance. ${ }^{(9)}$ The imperfect phase locking is seen as a coherence between the noise-induced switching between the wells and the deterministic intrawell motion. Phase locking has also received considerable attention in 
the biomathematics literature, namely in the context of periodically forced autonomous and integrate-and-fire-type (IF) oscillators. ${ }^{(10)}$ Gerstein and Mandelbrot ${ }^{(11)}$ briefly describe, in the context of the response of cat auditory fibers to short pulses (clicks), how the ISIH peaks of a periodically modulated random walk to an absorbing threshold fall at the integer multiples of the driving period. Glass and Mackey ${ }^{(12)}$ have studied phase locking in a simple integrate-and-fire model with the intent of explaining entrainment in higher functions such as respiratory control. A study of phase locking in a similar model in the presence of noise has shown that, between regions of phase space with stable phase-locking patterns, there are unstable zones with no phase locking. ${ }^{(13)}$ These unstable dynamics consist of quasiperiodic dynamics, arising for low-amplitude periodic input, and patterns with irregular skipped or intercalated beats at higher amplitude. Keener et al. ${ }^{(14)}$ have also carried out a very thorough study of the phase-locking dynamics of a (deterministic) leaky IF model with periodic forcing. The case of periodically forced cells which do not oscillate autonomously, such as the Purkinge fibers in the heart, ${ }^{(15)}$ has also received attention. In fact, Feingold et al. ${ }^{(16)}$ have shown, for an excitable system of the type considered here (though without noise), that phase-locking, quasiperiodic, and chaotic motion are possible, and that an Arnol'd tongue structure arises through the interaction of the external rhythm and an "effective intrinsic frequency" of the system. Finally, Alexander et $a l .{ }^{(17)}$ have investigated, using analytical and numerical techniques, the detailed phase-locking dynamics of the (deterministic) Fitzhugh-Nagumo equations (see Section 4).

Most of the aforementioned studies focus on the phase-locking patterns in the time domain, and the statistical distribution of the intervals between firings has received little attention, apart from brief discussions in refs. 11 and 13. We now consider interval distributions in a noisy bistable system, which will serve as a stepping stone toward the main focus of this paper, the existable model.

\section{MODELS OF NEURONAL BISTABILITY}

Landahl et al. ${ }^{(18)}$ were among the first to consider neurons as bistable devices driven by noise (see ref. 19 for a review of stochastic neural modeling). This simple description of the neuron attests simply to its main nonlinear property, namely the presence of a threshold which gates the influence of the input to the neuron on its output. Much theoretical and experimental work has been done on the effect that an external periodic forcing has on the switching behavior of a bistable system, especially in the context of stochastic resonance (see ref. 9 for a review). This leads in 
particular to the study of systems governed, in the limit of large damping, by the flow

$$
\frac{d x}{d t}=-\frac{d U(x)}{d x}+\xi(t)+m \sin (\omega t)
$$

where $U(x)$ is a double-well potential and $\xi(t)$ is a white Gaussian noise process of zero mean and correlation $\langle\xi(t) \xi(s)\rangle=2 D \delta(t-s)$. In what follows, the behavior of Eq. (1) is investigated numerically, with the process $\xi(t)$ replaced by an Ornstein-Uhlenbeck process governed by

$$
\frac{d \eta}{d t}=-\lambda \eta(t)+\lambda \xi(t)
$$

in order to have control over the correlation time $\tau_{c}=\lambda^{-1}$ of the noise. This Gaussian process has zero mean, and its autocorrelation is given by

$$
C(t, s)=C(|t-s|)=\langle\eta(t) \eta(s)\rangle=\left(D / \tau_{c}\right) \exp \left(-|t-s| / \tau_{c}\right)
$$

and thus its variance is $C(0)=D / \tau_{c}$. Various analytical results concerning mean first passage times (MFPT) and the distribution of residence times $T$ within a given well of the double-well potential $U(x)$ have been obtained. ${ }^{(9,20)}$

Bistable systems as elementary as the Schmitt trigger have been shown to exhibit the basic features of the biological data. ${ }^{(1,6,7)}$ These results may be summarized as follows. Denote the two states of the bistable system by $A$ and $B$, and assume that the amplitude of the periodic input is not sufficient to cause a state transition. In the absence of any noise, this system would remain confined to its initial state. In the presence of noise, state transitions will occur (with or without the periodic input), and the twostate output of the trigger will be a random telegraph signal. Distributions of intervals between these transitions are similar to those shown in Fig. 1 when an interval corresponds to the time between two successive $A \rightarrow B$ transitions (or $B \rightarrow A$ for a symmetric potential).

This description in which an interval is made up of two successive residence times is valid if both "states" of the neuron are locally stable as in the simple bistable model. The identification of "states" in the neural system is trickier, however, than for this simple model. It has been argued in ref. 1 that the typical excitable neuron has a characteristic currentvoltage relation which is $\mathrm{N}$-shaped, as is the flow associated with a bistable potential. One can thus identify the resting state of the neuron with the equilibrium point of the left well; both are stable in the absence of forcing (the cells of interest then simply fire spontaneously and randomly). The 
other state may correspond to an "excited state" (above threshold) which does not immediately reset to the resting state (certain excitable cells are known to exhibit such "long plateaus"--see ref. 21), to a "recovery state" characterized by hyperpolarization. In either case, a resetting event (which allows the vicinity of the resting state eventually to be reached) must occur between successive spikes. It is in this spirit that one is led to the association of interspike interval with a "back-and-forth" event in the bistable system.

Figure 2 is a plot of the ISIH for transition times generated by the standard quartic potential:

$$
\frac{d x}{d t}=x-x^{3}+m \sin (\omega t)+\eta(t)
$$

where $\eta(t)$ is again the Ornstein-Uhlenbeck process. The parameters are such that no deterministic switching occurs. The decay of the ISIH is exponential (data not shown). Very fast events are seen to occur (in the first bins), due to rapid successive crossings of the metastable state. At very high amplitude, the skipping behavior of the model becomes replaced by $1: 1$ firing. If the forcing amplitude or the noise intensity is too small, or the

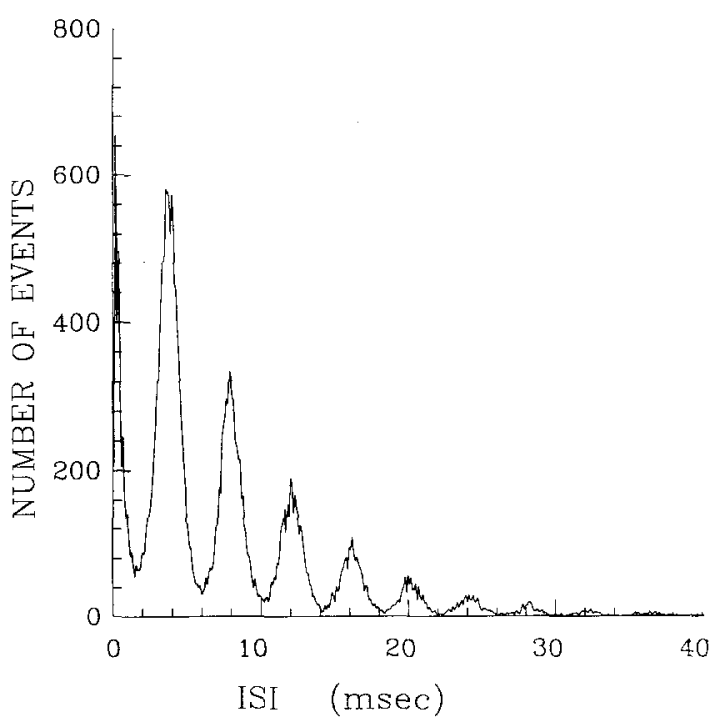

Fig. 2. ISIH obtained from the solution of the standard quartic system [Eq. (4)] with a forcing frequency of $250 \mathrm{~Hz}$. An interval corresponds to the time between successive $A \rightarrow B$ transitions. Parameters are $m=1.0, D=0.1, \tau_{c}=0.1, \omega=500 \pi$. 
forcing frequency is too high, "spikes" preferably occur every second or third cycle, each stimulus cycle bringing the particle closer to the barrier. In this case, the envelope decay is asymptotically exponential. ${ }^{(1,6)}$

\section{AN EXCITABLE MODEL}

The Hodgkin-Huxley equations ${ }^{(22)}$ capture the basic regenerative firing mechanisms of nerve cells. This model and certain lower-dimensional variants of it exhibit bistability between two fixed points over certain parameter ranges. ${ }^{(21)}$ They can also exhibit bistability between a fixed point and a limit cycle (periodic autonomous firing). Hence, in principle, they can be made to exhibit the phenomena of the simple bistable models. While it is possible that these kinds of bistability, in conjunction with periodic and stochastic forcing, underlie the skipping phenomenon, it would be interesting to find another mechanism which is perhaps more robust and typical of excitable models. We now investigate the behavior of a generic excitable cell model, the Fitzhugh-Nagumo (FHN) equations ${ }^{(22,23)}$ :

$$
\begin{aligned}
& \varepsilon \frac{d v}{d t}=v(v-a)(1-v)-\omega+\xi(t) \\
& \frac{d \omega}{d t}=v-d \omega-[b+r \sin (\beta t)]
\end{aligned}
$$

This system of two ODEs, which still has a cubic (or N-shaped) nonlinearity as in the model of the previous section, captures the basic mechanism of action potential generation. It automatically resets from the excited state to the resting state, which is not the case for the onedimensional bistable models investigated above. The FHN equations account for a recovery current which has the effect of hyperpolarizing the neuron membrane potential, i.e., making it temporarily more negative than the resting potential (with respect to the extracellular space), thus rendering the cell refractory to the generation of another spike for about a millisecond. This model allows, as do the $\mathrm{HH}$ equations [but not the models based on Eq. (1)], for repetitive firing in the absence of periodic forcing, through a supercritical Hopf bifurcation. ${ }^{(22)}$

The variable $v(t)$ is the fast "voltagelike variable," while $w(t)$ is the slower "recoverylike" variable. In Eqs. (5)-(6), the periodic forcing has been added to the dynamics of the recovery variable, while the stochastic forcing has been added to the dynamics of the voltage variable. The reason for this is to allow comparison with the deterministic picture, studied by 
Alexander et al., ${ }^{(17)}$ in which the periodic forcing is added to the slow dynamics. It is argued in ref. 17 that the transformation from

$$
\begin{aligned}
& \varepsilon \frac{d v}{d t}=v(v-a)(1-v)-\omega+A \sin (\beta t)+\xi(t) \\
& \frac{d \omega}{d t}=v-d \omega-b
\end{aligned}
$$

to Eqs. (5)-(6) is valid, and further holds in the singularly perturbed limit $\varepsilon \rightarrow 0$ as long as $\beta<1 / \varepsilon$. When $\beta$ is $O(1 / \varepsilon)$, the forcing cannot be isolated on the slow variable uniformly in $\varepsilon$; in fact, new phenomena arise when the frequency is comparable to the relaxation rate of the fast variable. The transformation from Eqs. (7)-(8) to Eqs. (5)-(6) can be accomplished by a change of variable. Consider the system

$$
\begin{aligned}
& \varepsilon \frac{d v}{d t}=p(v)-q(\omega, \phi(t))+\xi(t) \\
& \frac{d \omega}{d t}=g(v, w, \psi(t))
\end{aligned}
$$

If $\partial q / \partial \omega \neq 0$, then the change of variable $\tilde{\omega}=q(\omega, \phi)$ and its inverse $\omega=h(\tilde{\omega}, \phi)$ can be used, with help from the chain rule, to rewrite Eqs. (5)-(6) as

$$
\begin{aligned}
& \varepsilon \frac{d v}{d t}=p(v)-\tilde{\omega}+\xi(t) \\
& \frac{d \tilde{\omega}}{d t}=\frac{\partial q}{\partial \omega} g(v, h(\tilde{\omega}, \phi), \psi)+\frac{\partial q}{\partial \phi} \frac{d \phi(t)}{d t}
\end{aligned}
$$

In the case of interest here, $\phi(t)=I(t)=A \sin (\beta t), \tilde{\omega}=q=w-I(t)$, $\psi(t)=0$, and $g \equiv g(v, \omega)=v-d \omega-b$. The equation for the new recovery variable $\tilde{\omega}$ becomes

$$
\frac{d \tilde{\omega}}{d t}=v-d \tilde{\omega}-b-d I(t)-I^{\prime}(t)
$$

The term $-d I(t)-I^{\prime}(t)$ is equivalent to harmonic forcing such as $I(t)$ defined above when $A=r /\left(d^{2}+\beta^{2}\right)^{1 / 2}$. We have verified (data not shown) that, when the amplitudes in Eqs. (5)-(6) and Eqs. (7)-(8) are related by this expression, the numerical simulation results for the parameter ranges in our study are identical to within the expected statistical fluctuations. All the FHN simulations presented here are done on Eqs. (5)-(6). The com- 
plexity of this flow warrants the use of (digital) numerical simulations to investigate the spiking dynamics and stochastic resonance in the FHN equations. All our simulations are done using the Ornstein-Uhlenbeck noise [Eq. (2)] with correlation time set to 0.01 .

We focus our attention on whether the periodically forced FHN equations can exhibit an ISIH of the type shown in Fig. 1. It turns out that the answer is yes when parameters are such that the deterministic FHN is below but near the supercritical Hopf bifurcation at $b=b_{0}$, and that noise is added to the system as in Eqs. (5)-(6). A realization of this stochastic process is shown in Fig. 3. The ISIs are now computed from the times between positive-going crossings of a threshold, chosen as 0.5 , which is approximately half the peak height in the absence of noise. The peak structure of the ISIH becomes more visible when the stimulus modulates the bifurcation parameter $b$ [as in Eqs. (5)-(6)] in such a way that $b>b_{0}$ during part of the forcing cycle. ${ }^{(7)}$ Figure 4 plots the spontaneous ISIH for the FHN model, i.e., when the stimulus amplitude is zero. The ISIH has the shape of a gamma distribution, which also characterizes the experimen-

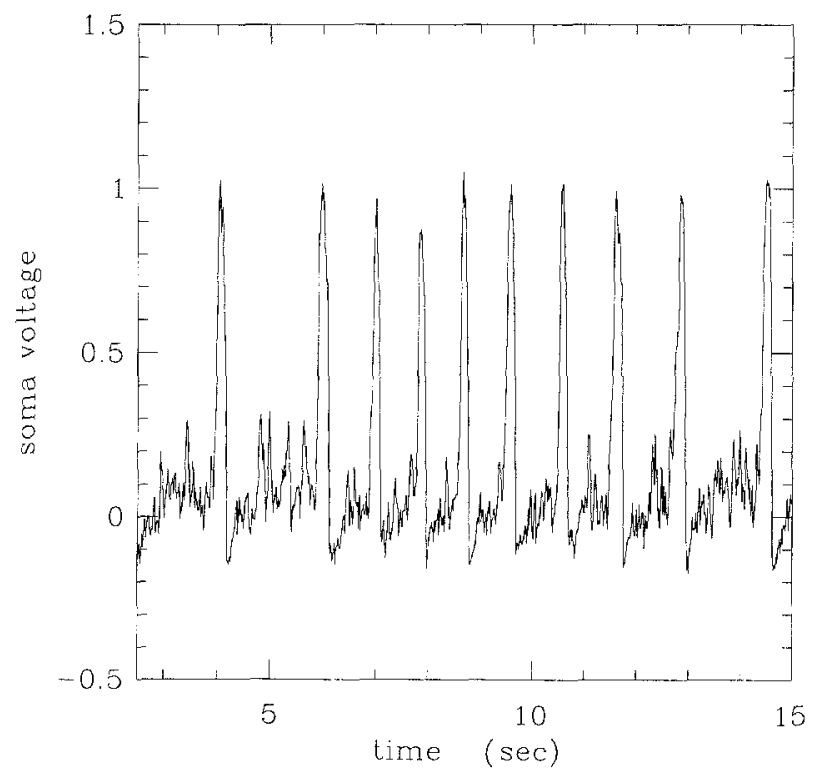

Fig. 3. A realization of the stochastic process given by Eqs. (4)-(5) corresponding to the Fitzhugh-Nagumo equations. The mean spiking period is $1.40 \mathrm{sec}$. The positive-going spikes are considered as action potentials when they reach a minimum amplitude (set at 0.5 in our study). Typical return maps are similar to that shown in Fig. 1a. Parameters are $\beta=15$, $a=0.5, b=0.15, d=1.0, \varepsilon=0.005, D=10^{-5}, \tau_{c}=0.01$, integrated using fourth-order RungeKutta with an integration time step of 0.0025 . 


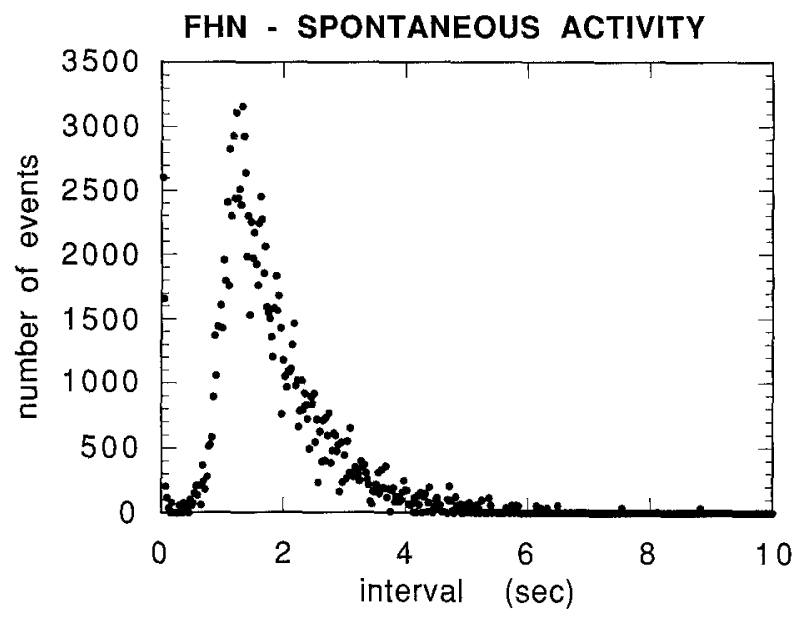

Fig. 4. Spontaneous ISIH for noisy FHN from which one can calculate mean first passage time to the threshold for action potentials, i.e., the mean firing rate (1.40). Parameters are $r=0.0, b=0.15$, and $D=10^{-5}$.
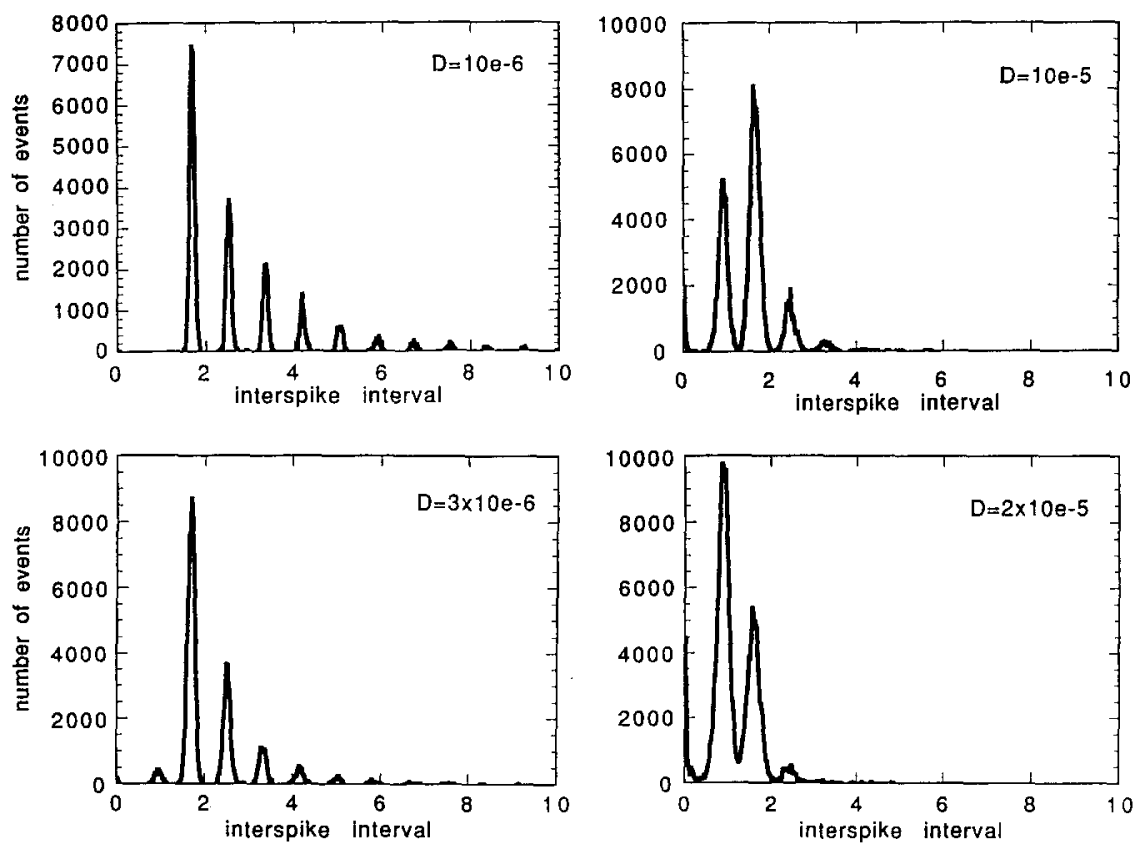

Fig. 5. ISIHs computed at four values of the noise intensity $D$. The forcing period is $0.84 \mathrm{sec}$ $(\beta=7.5), a=0.5, b=0.12, d=1.0, r=0.2$, and $\varepsilon=0.005$. Each ISIH is computed from 200 different realizations of the stochastic process, Eqs. (4)-(5), each one consisting of $5 \times 10^{6}$ integration time steps of 0.0025 (first 1000 time steps discarded as transients). 
tal spontaneous ISIHs. The bifurcation parameter is set at $b=0.15$, a value below the deterministic bifurcation point of $b_{0}=0.35$ for the parameters used in our study. The spontaneous ISIH is nevertheless relatively sharp, and becomes peaked toward the limit cycle period as $b$ increases (data not shown), owing to entrainment of the motion to the limit cycle.

The ISIHs are computed for four different noise intensities $D$ in Fig. 5 . Low noise intensities bring out many peaks, and the probability concentrates into the first peaks at the noise intensity increases. The ISIHs present the same features as the experimental ones, including the broad skirts on the peaks. At very large noise levels, the background stochastic firings dominate the ISIH (the space between the peaks fills in). The buildup of

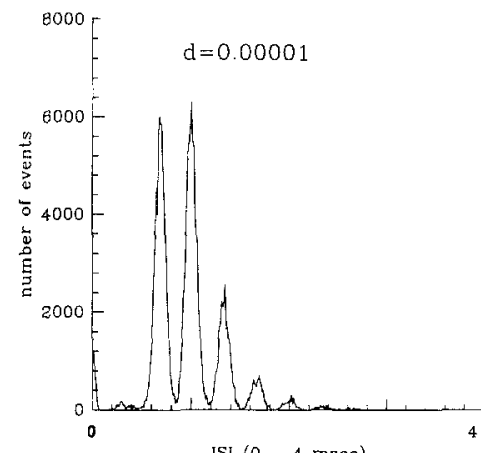

ISI $(0-4 \mathrm{msec})$

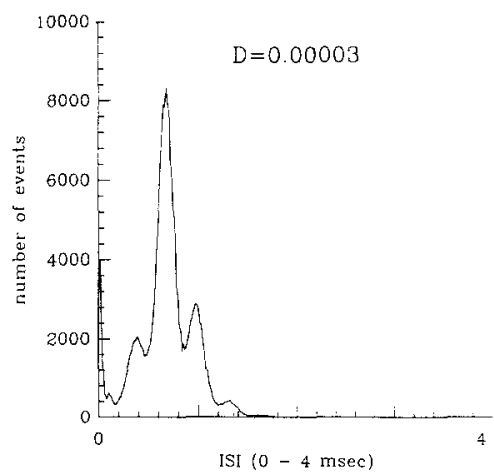

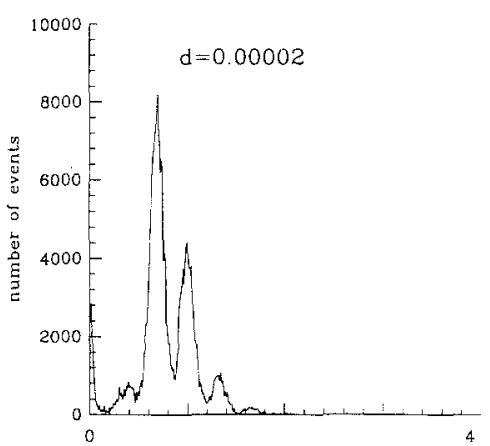

ISI $(0-4$ msec $)$

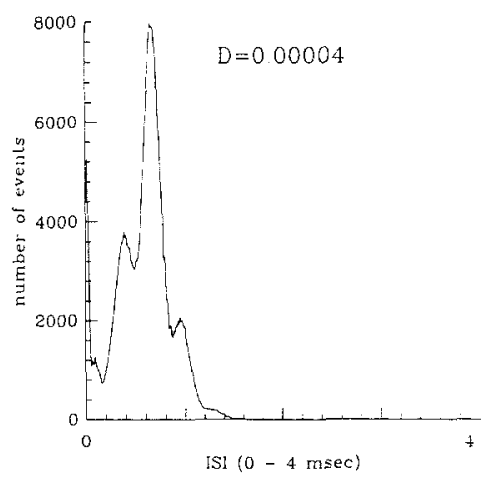

Fig. 6. ISIHs for the FHN equations at four values of the noise intensity with $\beta=15$. Equations (4)-(5) were integrated for $5 \times 10^{6}$ steps of $0.0025 \mathrm{sec}$, and this was repeated 20 times. The threshold for spiking is 0.5 . Other parameters are: $D=0.00001, t_{c}=0.01, a=0.5$, $b=0.15, c=1.0, d=1.0, \varepsilon=0.005$. The second peak (i.e., that centered at $2 T_{0}$ ) is seen to go through a maximum as the noise intensity is increased (see Section 5). 
the background noisy firing is also evident from the increasing peak widths with the noise intensity.

Figure 6 shows similar results, except that the frequency is now twice its value in Fig. 5 , and the bifurcation parameter is set closer to $b_{0}$. The interesting point here is that the first peak corresponding to the forcing period (0.42) appears suppressed, even at large noise intensity. In fact, the second peak (i.e., at $2 T_{0}$ ) always dominates, even though it goes through a maximum. The reason for this may be that the period of the limit cycle (which affects the dynamics for $b<b_{0}$ due to the noise), which is 0.77 , is
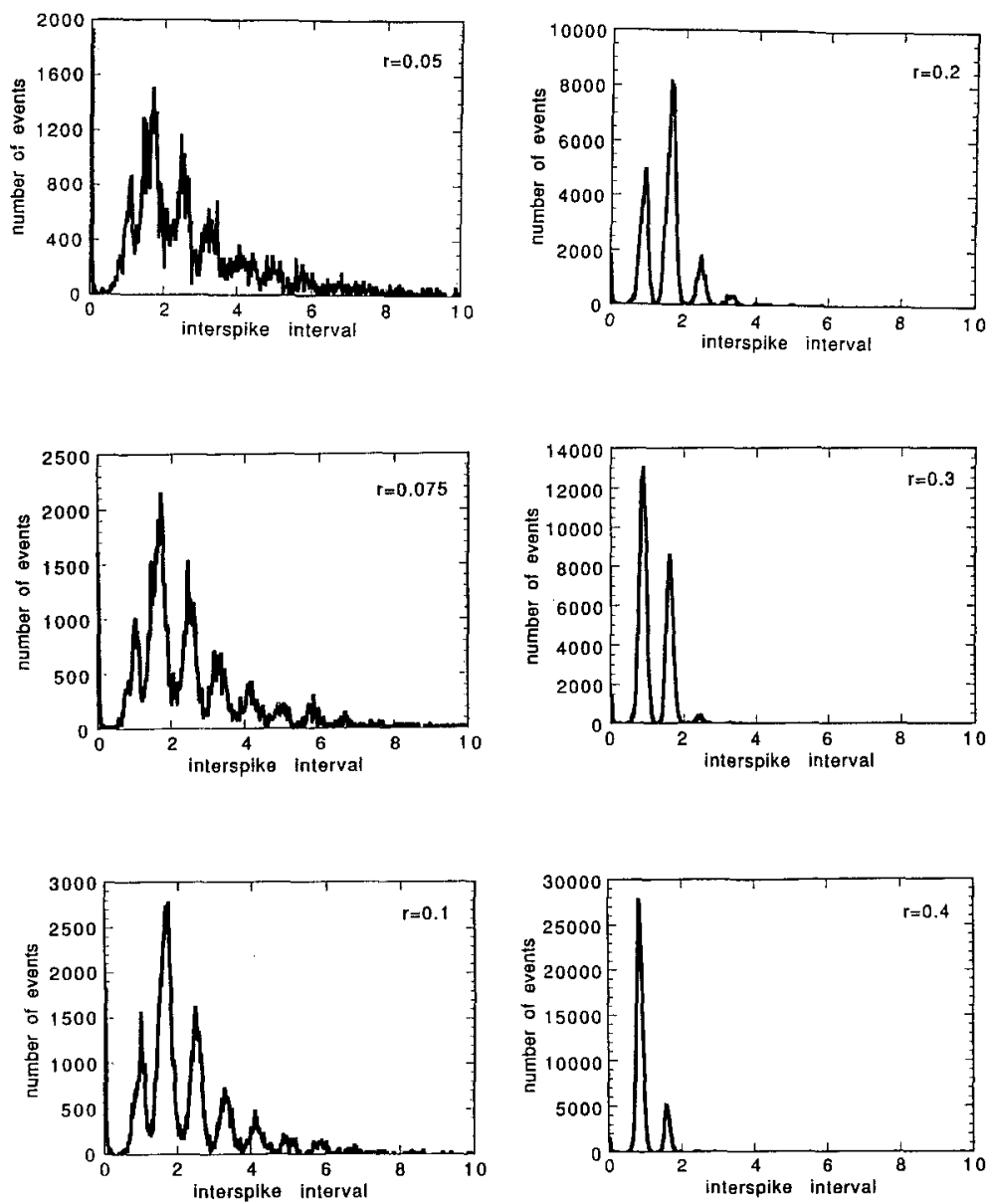

Fig. 7. ISIHs computed at six values of the stimulus amplitude $r$. Other parameters are $a=0.5, b=0.12, d=1.0, \beta=7.5$, and $\varepsilon=0.005$. Integration of Eqs. (4)-(5) is as in Fig. 5 . 
quite close to $2 T_{0}$; in other words, the dynamics entrains to the period of the noise-induced limit cycle, and skipping every other beat is a highly probable event. For large noise, the peak structure is seen to yield to the background stochastic firing in a clearer manner than in Fig. 5.

Figure 7 illustrates the effect of the stimulus amplitude on the ISIH. At low amplitude many noisy peaks are noticeable. As the amplitude increases, the rate of decay of the ISIH also increases, and the peaks become in fact narrower, a behavior seen in Fig. 5 for low noise levels. As the amplitude increases even further, the behavior tends to a 1:1 phase locking. Note finally that the ISIHs corresponding to low-amplitude stimuli and/or to high noise intensities exhibit many fast events, i.e., random high-frequency spiking.

\section{STOCHASTIC RESONANCE: INTERACTION OF THREE TIME SCALES}

Classical stochastic resonance (SR) occurs when the time scale imposed by the external periodic modulation becomes commensurate with an appropriately defined switching rate for the bistable system. Residence time histograms (RTHs) provide the natural context in which to investigate SR in our system. ${ }^{(1,6,7)}$ For bistable systems, the height of the second (or third, or fourth,...) peak goes through a maximum as a function of noise intensity. ${ }^{(20)}$ While this effect is a signature of a resonance, in the sense that the maximum implies a preferred switching interval, its precise relationship to SR as measured by the signal-to-noise (SNR) ratio has not been characterized completely. Many results indicate, however, that this resonance occurs at the same noise intensity as does that measured using the SNR (see, e.g., ref. 24).

Before addressing the issue of whether or not the excitable FHN model exhibits SR, it is important to understand in what sense (if any) the model is bistable. The Hopf bifurcation in the FHN equations is supercritical, implying no bistability, even though the limit cycle amplitude increases abruptly, reminiscent of a subcritical bifurcation. However, there is a noise-induced limit cycle. Thus, in some sense, the bistability can be seen as arising between a fixed point (the resting potential) and an oscillatory state which produces spikes at a mean rate dependent on the noise, in analogy to bistability between a fixed point and a repetitively firing state seen in some neuron models. ${ }^{(21)}$ SR would then occur when the mean time the voltage takes to go from the resting value to the threshold for spiking in the presence of noise becomes commensurate with the forcing period. This situation is also analogous to that studied by Fletcher et al. ${ }^{(25)}$ 
in which an escape time to an absorbing threshold for a particle undergoing a random walk in some potential becomes minimal in the presence of a commensurate periodic modulation. The "neural mean first passage time" to the threshold can be evaluated simply as the mean of the ISIH (MFPTs for neuron models are discussed in ref. 19).

The behavior of the peak heights as a function of $D$ and $r$ is shown in Figs. 8 and 9, respectively. While the height of the first peak is seen to increase monotonically with $D$ or $r$, the second peak goes through a maximum, as observed by Zhou et al. ${ }^{(20)}$ for the single-well RTHs. However, the first peak can go back down at large $D$ (not shown), suggesting that SR may occur at the maximum of this peak. This further depends on what one measures, since the position of the first peak will be affected the most by the growing background firing distribution on which it becomes superimposed (see also ref. 26). The second peak achieves its maximum value (as a function of $D$ ) well before it is reached by the first peak. It is possible that, by the time the first peak dominates the others, much of the coherence between the input and the firings may be lost, since the high noise level causes the switchings to occur over a whole range of times during each half cycle of the stimulus. ${ }^{(27)}$ Thus, as for bistable systems, the coherence between signal and switching in the FHN model may be maximum when the second peak is maximum.

We are also confronted with two different views of resonance: that in which the peaks are unnormalized, and that in which they are. We have

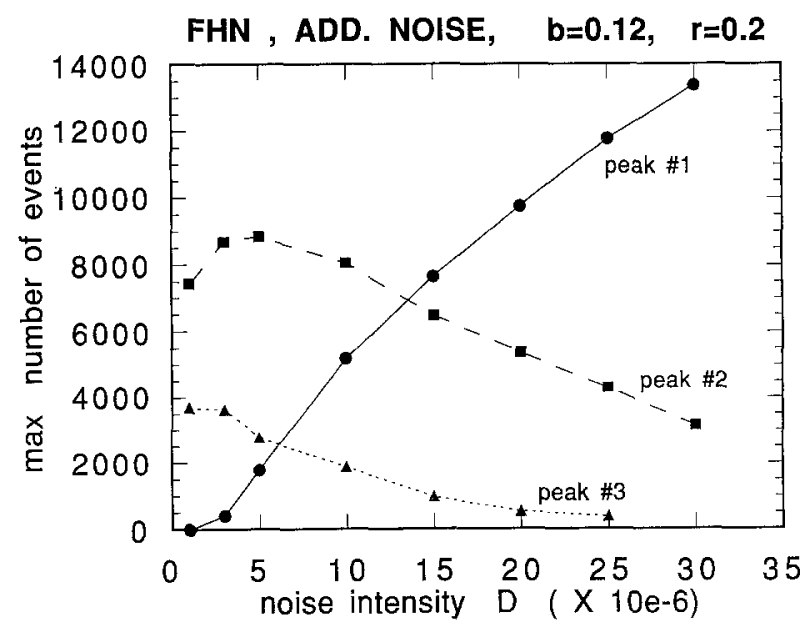

Fig. 8. Height of the first three peaks in the ISIHs of Fig. 5 as a function of noise intensity $D$. 


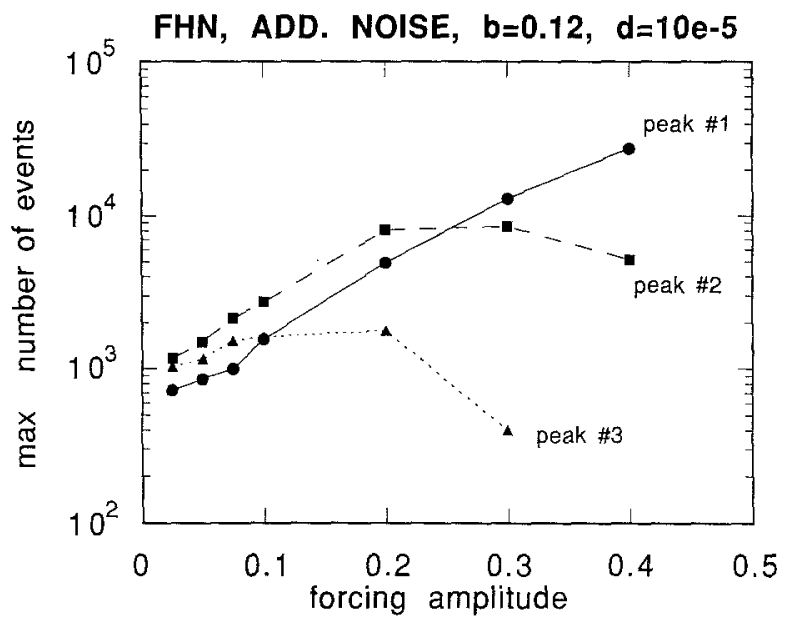

Fig. 9. Height of the first three peaks in the ISIHs of Fig. 7 as a function of forcing amplitude $r$. Note the exponential increase of the first and second peaks at low amplitude.

found for the parameter range investigated (data not shown) that if our ISIHs are normalized, Figs. 8 and 9 are qualitatively different. Also, if the number of events in the bins corresponding to $T_{0}$ and $2 T_{0}$ were used, regardless of whether these bins correspond to peak maxima, Fig. 8 would then show the second peak decreasing monotonically and the first peak going through a maximum. One can have the situation where one peak has less probability at one value of $D$ than at another, even though in absolute numbers it has more events, all simulations lasting the same number of stimulus cycles. So even though, relatively speaking, the events in this peak are less probable than at the other noise value, more firings take place, and more information about the stimulus appears conveyed to the neuron's output. The area under the peak may then be the important quantity. One hopes that the issues will be settled by future investigations.

The mean of the ISIH for the FHN system is about 1-1.5 sec when $D \approx 10^{-5}$ (see Fig. 4 for $b=0.15$; similar values were obtained for $b=0.12$ used for the ISIH computation with $r \neq 0$ ). The stimulus frequencies used are 1.2-2.4 (corresponding to $\beta=7.5-15$ ). Hence we are already in a range where resonance can in principle occur, if it occurs by a mechanism similar to that in one-dimensional bistable systems. A maximum in the height of the second peak of the ISIH is in fact seen for $D \approx 10^{-5}$. The presence of the third time scale, i.e., the limit cycle, implies that as the model operates closer to its Hopf bifurcation point, the mean of the spontaneous activity becomes strongly determined by the limit cycle period. The mean firing time being close to this period, resonance will ensue especially when the 
neuron is stimulated at this frequency, which is a preferred frequency for the system. Thus, as the vicinity of the bifurcation point is approached, the description shifts from stochastic resonance to normal resonance since the FHN equations behave as an underdamped oscillator driven by noise.

\section{DISCUSSION}

The motivation for this work is to understand the generic properties of bistable and excitable models with respect to the encoding of sensory information, and in particular to stochastic resonance. The connection between the biology and the models is made plausible by identifying the fixed points of the flow with those derived from the N-shaped currentvoltage relation of the neurons. The models studied here reproduce the data when their parameters are such that deterministic switching is minimal or nonexistent. Hence the noise is necessary to transfer the signal from the neuron's input to its output firing activity.

Another possibility is that the ISIHs arise out of chaotic behavior of the neurons, corresponding to chaotic solutions of Eqs. (5)-(6) known to occur for certain parameters. An ISIH built from such a chaotic solution is shown in Fig. 10a, along with its return map in Fig. 10b. It certainly does not reproduce the data as convincingly as the models considered up to now. The peaks have a different shape and do not line up very well with integer multiples of the driving period. Further, a slight parameter change can drastically modify the ISIH, as a different chaotic solution or even a simple or complicated phase-locked solution can arise. Additive noise does smoothe out the ISIH and the return map, as shown in Figs. 10c and 10d, but again a small change, e.g., in frequency can yield quite a different picture. This sensitivity of the ISIHs to small parameter variations was not present in the bistable nor in the excitable models studied here. On the other hand, it appears difficult to obtain ISIHs for the FHN system with many peaks when the first peak is the highest; this behavior is most common in the (auditory) neural data, and easily reproduced by the bistable model. Possible reasons for this are: (1) the frequencies investigated are too high; (2) there is entrainment to the intrinsic period of the limit cycle which lies nearby in parameter space; and (3) the mechanism for skipping is not the one studied here. It is possible, for example, that a good or better fit to the data can result from operating the FHN model in a parameter range where one has many periodic phase-locked solutions in close proximity (and possibly multistability between them) rather than chaos or a noise-induced limit cycle. ${ }^{(26)}$ Results concerning this point will be reported elsewhere.

Noiseless neural models typically have a very limited dynamic encoding range. If a constant stimulus is subthreshold, no spikes occur; if it is 

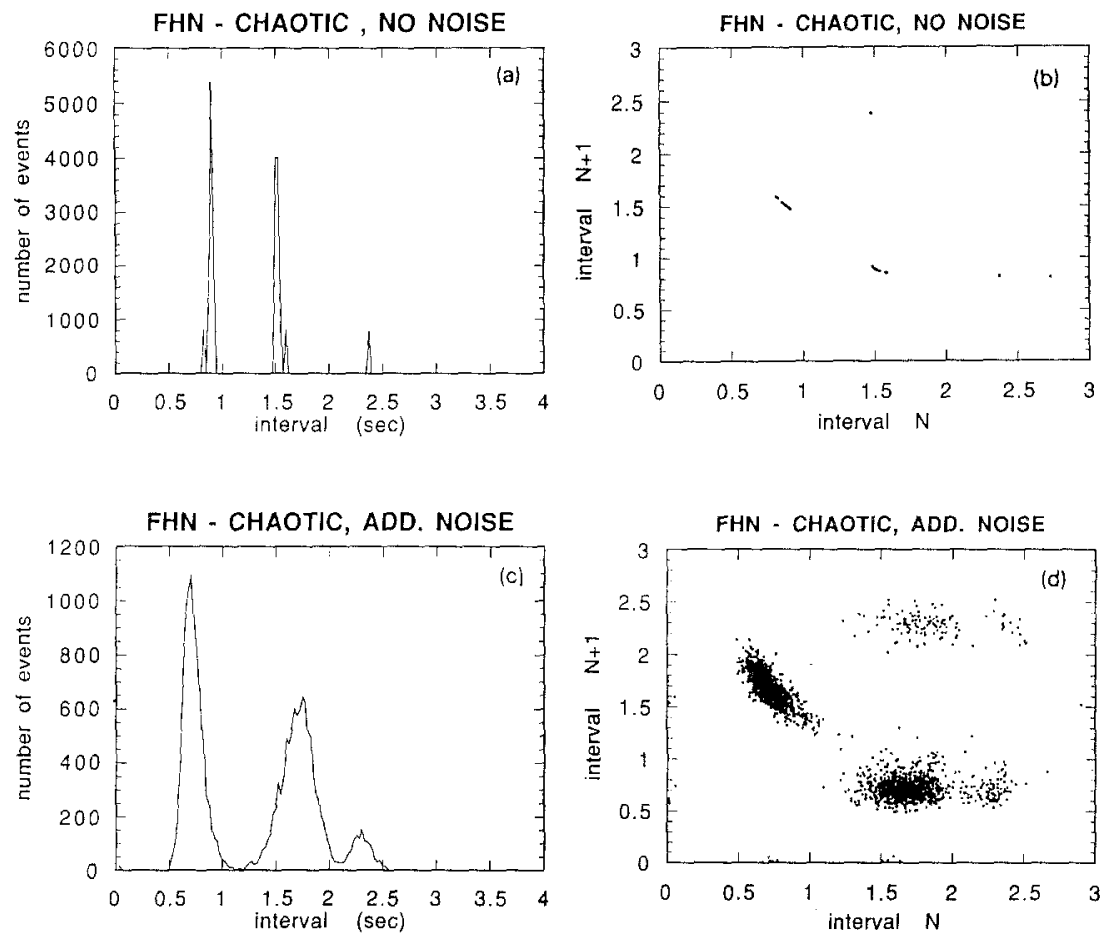

Fig. 10. ISIHs and interval return maps obtained from the FHN equations in the chaotic regime, with and without external additive noise. (a) ISIH, no noise, and (b) corresponding return map, no noise; (c) ISIH, $D=10^{-5}$, and (d) corresponding return map. Parameters are $a=0.5, b=0.2, d=1.0, r=0.2, \varepsilon=0.005, \beta=2.625$.

suprathreshold, firing at a constant and usually high rate occurs. This rate does not depend sensitively on the value of stimulus. Various authors ${ }^{(28,29)}$ have shown that noise can linearize the firing-frequency vs. stimulusamplitude relation of the neuron, thereby increasing the dynamic encoding range. Although it is still far from clear whether the encoding uses mean spike rate, the ISIH, or the precise timing of the ISIs in a spike train, it is interesting that the linearization property arises naturally in the neuron models considered in this work.

It is interesting that there is practically no predictability, linear or nonlinear, in ISI data of the type considered here. ${ }^{(7)}$ Hence, one is tempted to conclude that the temporal features of the data are not so important as its statistical features, reflected namely in the ISIH. However, there is evidence (for the auditory fibers at least) for clustering and long-term correlations in the data, exhibited through Fano-factor time curves. ${ }^{(8)}$ Finding the proper dynamical model then requires proper consideration of the temporal as 
well as statistical properties of the spiking. Finally, stochastic resonance in the context of residence time histograms seems to depend on the way one measures it. Theoretical work is still needed to clarify the relation between SR and the peak heights, as well as the sense in which there is resonance.

\section{ACKNOWLEDGMENTS}

The author acknowledges useful discussions with Dante Chialvo, Frank Moss, Adi Bulsara, Valerie Gremillion, Vania Apkarian, Ralph Siegel, and William Rhode. Many thanks are due to W. Rhode for providing the data. Support from Los Alamos National Laboratory and NSERC (Canada) in the form of postdoctoral fellowships is also acknowledged. This work was done under the auspices of the National Institute of Mental Health through grant 1-R01-MH47184-01.

\section{REFERENCES}

1. A. Longtin, A. Bulsara, and F. Moss, Time interval sequences in bistable systems and the noise-induced transmission of information by sensory neurons, Phys. Rev. Lett. 67:656 (1991).

2. J. Rose, J. Brugge, D. Anderson, and J. Hind, Phase-locked response to low frequency tones in single auditory nerve fibers of the squirrel monkey, J. Neurophysiol. 30:769 (1967).

3. W. Talbot, I. Darian-Smith, H. Kornhuber, and V. Mountcastle, The sense of fluttervibration: Comparison of the human capacity with response patterns of mechanoreceptive afferents for the monkey hand, J. Neurophysiol. 31:301 (1968).

4. T. Ogawa, P. O. Bishop, and W. R. Levick, Temporal characteristics of responses to photic stimulation by single ganglion cells in the unopened eye of the cat, J. Neurophysiol. 6:2 (1966).

5. R. M. Siegel, Non-linear dynamical system theory and primary visual cortical processing, Physica 42D:385 (1990).

6. A Longtin, A. Bulsara, D. Pearson, and F. Moss, J. Neuroscience, submitted.

7. A. Longtin, Deterministic and stochastic dynamics of periodically forced neurons, Center Nonlinear Studies Newsl. 74:1 (1992) (Los Alamos National Laboratory preprint LA-UR-92-163).

8. M. C. Teich, Fractal neuronal firing patterns, in Single Neuron Computation (Academic Press, New York, 1992), p. 589.

9. F. Moss, Stochastic resonance: From the ice ages to the monkey's ear, in Some Problems in Statistical Physics, G. H. Weiss, ed. (SIAM, Philadelphia, 1992).

10. L. Glass and M. C. Mackey, From Clocks to Chaos. The Rhythms of Life (Princeton University Press, Princeton, New Jersey, 1988).

11. G. L. Gerstein and B. Mandelbrot, Random walk models for the spike activity of a single neuron, Biophys. J. 4:41 (1964).

12. L. Glass and M. C. Mackey, A simple model for phase locking of biological oscillators, J. Math. Biol. 7:339 (1979). 
13. L. Glass, C. Graves, G. A. Petrillo, and M. C. Mackey, Unstable dynamics of a periodically driven oscillator in the presence of noise, J. Theor. Biol. 86:455 (1980).

14. J. P. Keener, F. C. Hoppensteadt, and J. Rinzel, Integrate-and-fire models of nerve membrane response to oscillatory input, SIAM J. Appl. Math. $41: 503$ (1981).

15. D. R. Chialvo and J. Jalife, Non-linear dynamics in cardiac excitation and impulse propagation, Nature 330:749 (1987).

16. M. Feingold, D. L. Gonzalez, O. Piro, and H. Viturro, Phase locking, period doubling, and chaotic phenomena in externally driven excitable systems, Phys. Rev. 37A:4060 (1988).

17. J. C. Alexander, E. J. Doedel, and H. G. Othmer, On the resonance structure in a forced excitable system, SIAM J. Appl. Math. 50:1373 (1990).

18. H. Landahl, W. S. McCulloch, and W. Pitts, A statistical consequence of the logical calculus of nervous nets, Bull. Math. Biophys. 5:135 (1943).

19. H. C. Tuckwell, Stochastic Processes in the Neurosciences (SIAM, Philadelphia, 1989).

20. T. Zhou, F. Moss, and P. Jung, Escape-time distributions of a periodically modulated bistable system with noise. Phys. Rev. A 42:3161 (1990).

21. J. Rinzel and G. B. Ermentrout, Analysis of neural excitability and oscillations, in Methods in Neuronal Modeling, C. Koch and I. Segev, eds. (MIT Press, Cambridge, Massachusetts, 1989).

22. J. Cronin, Mathematical Aspects of Hodgkin-Huxley Neural Theory (Cambridge University Press, New York, 1987).

23. R. A. Fitzhugh, Impulses and physiological states in theoretical models of nerve membrane, Biophys. J. 1:445 (1961).

24. L. Gammaitoni, M. Martinelli, L. Pardis, and S. Santucci, J. Stat. Phys. 70:425 (1993).

25. J. Fletcher, S. Havlin, and G. H. Weiss, First passage time problems in time-dependent fields, J. Stat. Phys. 51:215 (1988).

26. D. R. Chialvo and A. V. Apkarian, J. Stat. Phys. 70:375 (1993).

27. B. McNamara and K. Wiesenfeld, Theory of stochastic resonance, Phys. Rev. A 39:4854 (1989).

28. H. Treutlein and K. Schulten, Noise induced limit cycles of the Bonhoeffer--Van der Pol model of neural pulses, Ber. Bunsenges. Phys. Chem. 89:710 (1985).

29. X. Yu and E. R. Lewis, Studies with spike initiators: Linearization by noise allows continuous signal modulation in neural networks, IEEE Trans. Biomed. Eng. 36:36 (1989). 